\title{
A low-cost particle counter and signal processing method for indoor air pollution
}

\author{
M. D. Taylor \& I. R. Nourbakhsh \\ The Robotics Institute, Carnegie Mellon University, USA
}

\begin{abstract}
Indoor air quality is closely linked with respiratory and cardiovascular health, prompting a need for affordable home air quality monitors. The newly-developed Speck is a very-low-cost indoor monitor for measuring fine particulate matter using optical sensors and a unique data processing algorithm. In this paper, we examine the performance of the Speck alongside two professional handheld particle counters (one HHPC-6 and one HHPC-6+) in household environments during cooking events and incense burning events. We demonstrate $r^{2}$ correlation values during the cooking event of greater than 0.98 between each pair of Specks and greater than 0.92 between each Speck and $2 \mu \mathrm{m}$ particle counts from the HHPC$6 / 6+$ monitors. The error between the Specks and the HHPC-6+ $2 \mu \mathrm{m}$ channel is less than the error between the HHPC-6 and HHPC- $6+2 \mu \mathrm{m}$ channels. The incense test yielded weaker correlation values, possibly due to uneven distribution of the smoke across the test setup. The distribution of particle sizes appears to be approximately the same as that generated from cooking. We conclude from these experiments that the Speck exhibits a strong correlation with professional particle counters, and that the error between the Speck and one professional unit is comparable to or less than the error between two very similar professional units. Keywords: $P M_{2.5}$, particulate matter, air quality monitoring, low cost sensors, calibration, indoor air quality.
\end{abstract}

\section{Introduction}

Fine airborne particles smaller than 2.5 microns, collectively designated as $P M_{2.5}$, pose a serious health risk to the public. These airborne particulates have been linked to significantly increased risk of cardiopulmonary and respiratory illnesses, particularly in the elderly and other sensitive populations [1-3]. 
$P M_{2.5}$ can be composed of any number of airborne particulate substances, and is a particularly useful metric in predicting adverse health effects [4]. Outdoor components can commonly include products from combustion such as diesel exhaust and coal particulates [5] but not larger particles such as pollen. Indoor sources are often present as well, including fine dust and particulates created from certain kinds of cooking, such as frying.

Because $P M_{2.5}$ is a measurement that can indicate the presence of a diverse range of pollutants [6], it is an ideal single metric for personal air quality monitors. Unfortunately, existing particle counters are almost solely available in the scientific and industrial markets, and are much more expensive than is practical for a home budget. The Speck is a low-cost $P M_{2.5}$ monitor designed for individual use in homes and in the workplace.

Related works, including [7], have established that inexpensive sensors designed to detect larger dust particles can also be used to detect particles in the $2 \mu \mathrm{m}$ range. Typically, these experiments use a moving average of raw sensor readings, with a linear scale factor used to match these values to particle counts or $2 \mu \mathrm{m}$ values from a professional monitor.

There are a small number of personal air quality monitors available on the market. The Air Quality Egg [8], available from Wicked Device, uses a similar dust sensor to those cited in [7] in its particulate sensor add-on. The Egg and daughterboard, at a combined cost of $\$ 243$, upload data to a publicly-available map currently populated with 1094 units. The Dylos [9], priced from $\$ 199$ to $\$ 425$, is widely used in experiments and publications where inexpensive and often mobile particulate monitoring is desired. The basic Dylos units use custom laser optics and report particle counts larger than $2.5 \mu \mathrm{m}$ and larger than $0.5 \mu \mathrm{m}$ on two separate channels. Advanced models feature additional functions such as additional size channels, datalogging capability, and battery power. In China, where air quality is a major concern within cities, several low-cost particulate monitors are available, though these have not been as extensively characterized or used in air quality
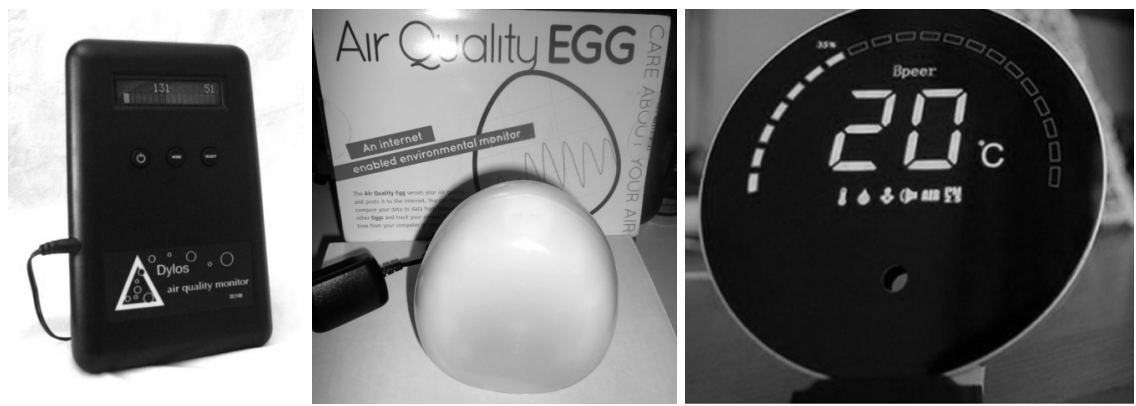

Figure 1: From left to right, the Dylos [9], Air Quality Egg [8], and BPEER [10] instruments, all commercially available. 
studies in comparison with the Dylos. These include the BPEER [10] and Air.Air! [11] devices, both of which rely on ambient airflow through a central passage.

\section{Speck}

The Speck utilizes an inexpensive DSM501a dust sensor [12] rather than custom optics, but also employs a small fan to increase airflow. The Speck contains on-board signal processing and storage in addition to a color LCD touchscreen for the user interface. Power is supplied via USB, and data can be downloaded directly to any computer. The interface allows users to view the current estimate of $2 \mu \mathrm{m}$ particle concentration as well as a scaled estimate of $P M_{2.5}$ in $\mu \mathrm{g} / \mathrm{m}^{3}$. The interface can also graph the past hour or past 12 hours of data on-screen, allowing for quick access to historical data.

The output of the DSM501a dust sensor is a digital pin which is pulled low when particles are detected in the optical chamber. According to the datasheet, the duty cycle is approximately proportional to the number of detected particles. The period of the sensor varies greatly, however, especially at low particle concentrations. While the duration of a low pulse (indicating detected particles) rarely exceeds $100 \mathrm{~ms}$, the duration between pulses can last from under one second to more than one minute. We observe that single-cycle readings are too noisy to be used directly. Instead, our algorithm samples the sensor 10,000 times per second, and uses the number of low samples each second as an input to an asymmetric filtering function. This input is herein referred to as the raw sensor value.

$$
e_{t+1}= \begin{cases}\left(A * \text { raw }_{t}-e s t_{t}\right) / B+e s t_{t} & : \operatorname{raw}_{t}>0 \\ (1-D) * e s t_{t} & : \operatorname{raw}_{t}=0\end{cases}
$$

The piecewise function given in eqn (1) describes the second-by-second processing of the raw sensor values, where $e^{s} t_{t}$ is the Speck's $2 \mu \mathrm{m}$ particle count estimate at time $t, \operatorname{raw}_{t}$ is the raw sensor value at time $t$, and $A, B$, and $D$ are constants. We observe that the individual raw sensor values are frequently zero in all but visibly smoky environments, though the non-zero values tend

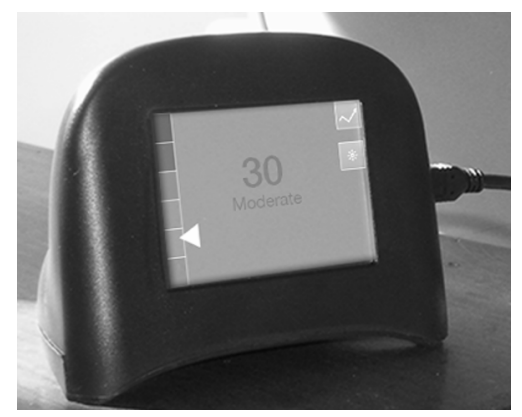

Figure 2: The Speck air quality monitor. 


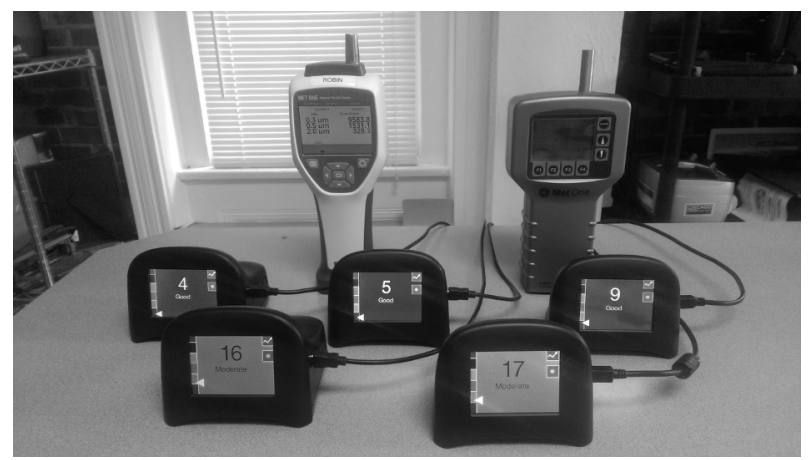

Figure 3: Calibration setup showing five Specks, the HHPC-6+ (left), and the HHPC-6 (right). Displayed values are scaled down from recorded ppl counts.

to increase linearly with particle concentration. Because of this, we design our filtering algorithm to give more weight to non-zero raw values. Each second, if the raw value is non-zero, we increment or decrement our current estimate at a rate proportional to the difference between the estimate and the raw value scaled by a constant. If the raw value is zero, the estimate exponentially decays toward zero at a lower rate. The resulting behavior is that the estimate quickly responds to non-zero raw values, but decays toward zero slowly due to the potential for long pauses between pulses.

The constants in eqn (1) were empirically determined in previous experiments through post-processing optimization of raw data from a single prototype Speck. The cost function selects values for the best fit of the estimate against the $2 \mu \mathrm{m}$ channel of the HHPC-6.

As we have since discovered, there is some variation between sensors such that multiple uncalibrated Speck units may display different values when co-located. The following experiments were performed to determine whether accuracy and precision can be achieved by linearly scaling the estimate using a constant unique to each Speck unit.

\section{Methods}

\subsection{Calibration pre-test}

The two primary experiments presented in this paper are preceded by a basic calibration test used to select and adjust three Specks. We begin with five Speck units running in parallel with one HHPC-6+ particle counter and one HHPC-6 particle counter. The HHPC units log particle counts within six size ranges. The HHPC-6 unit measures $0.3 \mu \mathrm{m}, 0.5 \mu \mathrm{m}, 0.7 \mu \mathrm{m}, 1 \mu \mathrm{m}, 2 \mu \mathrm{m}$, and $5 \mu \mathrm{m}$ sizes, while the newer HHPC-6+ unit measures $0.3 \mu \mathrm{m}, 0.5 \mu \mathrm{m}, 1 \mu \mathrm{m}, 2 \mu \mathrm{m}, 5 \mu \mathrm{m}$, and $10 \mu \mathrm{m}$ 


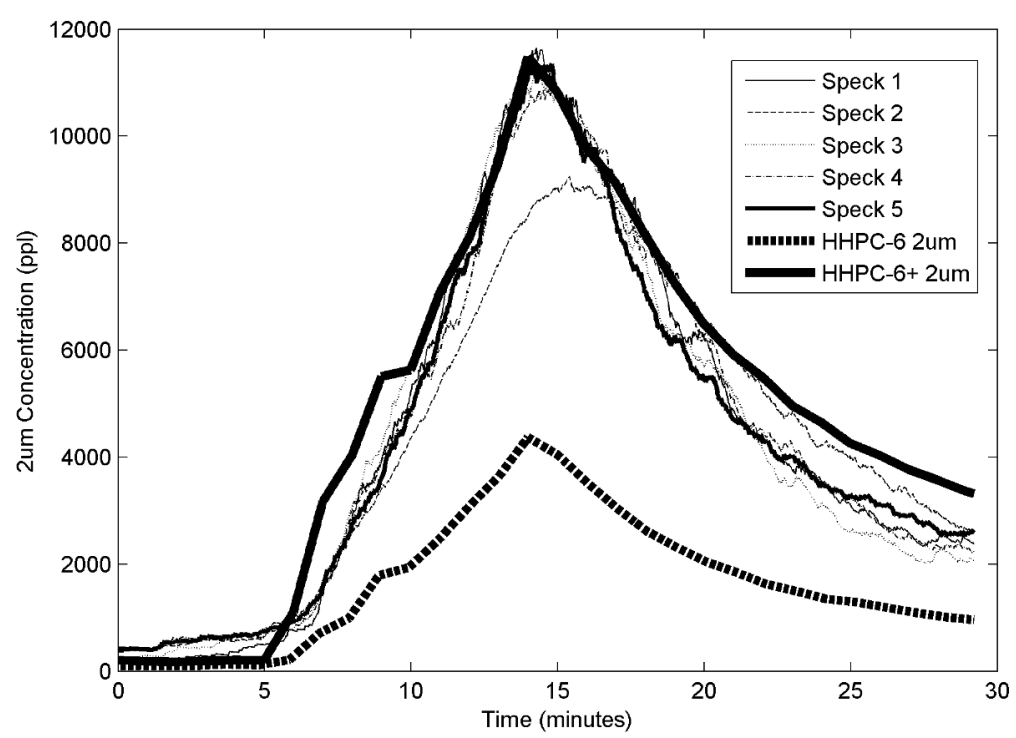

Figure 4: Plot of calibration data over time. Note the large discrepancy between the HHPC-6 channels.

sizes. We expose these seven instruments to one half-hour cooking event in order to gather an appropriate calibration dataset with high dynamic range. The cooking event involves frying poppadam (Indian lentil crackers) in a medium-sized kitchen with windows closed and the stove hood off. After approximately 15 minutes, we cease cooking and allow the air to clear naturally.

Our basic calibration involves scaling each of the Speck instrument outputs in order to minimize the mean percent error between the Speck signals and the $2 \mu \mathrm{m}$ channel of the HHPC-6+. This minimization is given in eqn (2), where $C$ is the calibration constant and $H_{t}$ is the HHPC- $6+2 \mu$ m measurement at time $t$. Of the five Specks, we select the three with the highest inter-Speck $r^{2}$ values (given in table 1) for use in subsequent tests, specifically Specks 1, 4, and 5 from this calibration set.

$$
\min _{C} \frac{\left|C * e s t_{t}-H_{t}\right|}{H_{t}}
$$

\subsection{Cooking test}

In the cooking test, the three most consistent Specks and the two HHPC particle counters tests are exposed to a second cooking test, with environmental conditions similar to those used in the calibration pre-test. The outputs of the Specks are scaled by the calibration values calculated in the pre-test. Figure 5 shows that the 
Table 1: Table of pairwise $r^{2}$ values for all Specks for the calibration test.

\begin{tabular}{|l|l|l|l|l|l|}
\hline & Speck 1 & Speck 2 & Speck 3 & Speck 4 & Speck 5 \\
\hline Speck 1 & 1 & 0.935 & 0.991 & 0.991 & 0.993 \\
\hline Speck 2 & 0.935 & 1 & 0.902 & 0.955 & 0.922 \\
\hline Speck 3 & 0.991 & 0.902 & 1 & 0.979 & 0.985 \\
\hline Speck 4 & 0.991 & 0.955 & 0.979 & 1 & 0.985 \\
\hline Speck 5 & 0.993 & 0.922 & 0.985 & 0.985 & 1 \\
\hline
\end{tabular}

Table 2: Table of scalar calibration values.

\begin{tabular}{|l|c|}
\hline & Calibration constant \\
\hline Speck 1 & 4.09 \\
\hline Speck 2 & 1.19 \\
\hline Speck 3 & 2.92 \\
\hline Speck 4 & 2.78 \\
\hline Speck 5 & 3.21 \\
\hline
\end{tabular}

calibrated Specks perform similarly in magnitude and shape to the HHPC- $6+2 \mu \mathrm{m}$ channel. Additionally, table 3 shows that the Speck units correlate strongly with the $2 \mu \mathrm{m}$ channels for both HHPC instruments. While the channels of the two HHPC units correlate strongly to one another, there is a significant discrepancy between the magnitudes of the HHPC- 6 and the HHPC-6+ measurements, shown in figure 6.

\subsection{Incense test}

The same three Specks and two HHPC particle counters are then placed in a small, closed room, where incense is burned for approximately five minutes, followed by a 45 minute rest period, and another five minute burning period. Ten minutes after the second burning, a HEPA air purifier is turned on until particle counts have approached the initial baseline. In this experiment, the Specks underestimated the $2 \mu \mathrm{m}$ particle concentration with respect to the HHPC-6+, as shown in figure 8 . 
The correlation values between all five instruments in table 4 were notably less than those from the cooking experiment. Despite the weaker correlation between HHPC instruments, the magnitude of the errors between these instruments is similar to those in the cooking experiment.

\section{Results and discussion}

In both the calibration pre-test and the cooking test, we observe that the pairwise $r^{2}$ value between every Speck unit exceeds 0.90 , and exceeds 0.98 for all but one unit. This demonstrates that the Speck design is internally consistent in its ability to detect pollutants from cooking. Furthermore, the $r^{2}$ values between the three test Specks exhibit a similarly strong correlation with the $2 \mu \mathrm{m}$ channels from the HHPC-6 and HHPC-6+ instruments, as well as the $1 \mu \mathrm{m}$ channel from the HHPC-6. This suggests that the Specks are most capable of detecting particles in the $2 \mu \mathrm{m}$ range.

The two HHPC monitors show strong correlation values between their corresponding size channels during the cooking test, which demonstrates the instruments' capability to detect the same particle signals. We also note the strong correlation between the HHPC-6 $1 \mu \mathrm{m}$ and $2 \mu \mathrm{m}$ channels, which may indicate a possible overlap in detection for these two sizes on this instrument. Though not as strong, the correlation is also high between these two channels on the HHPC$6+$ instrument, which could also indicate that the ratio between these two particle

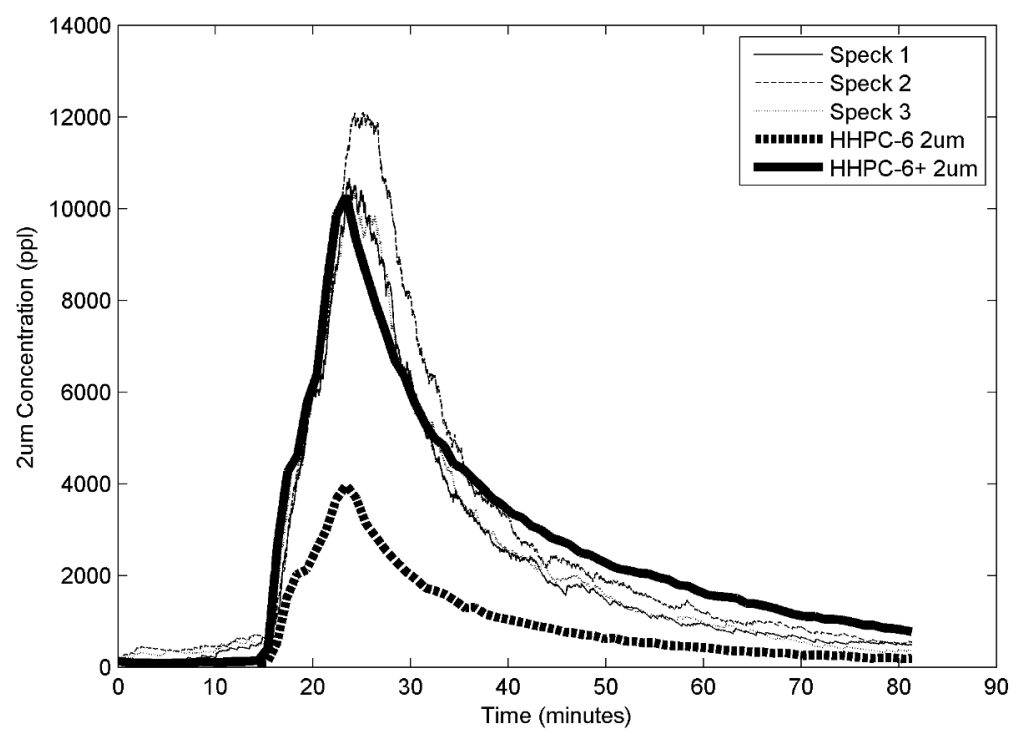

Figure 5: Plot of cooking data over time. 

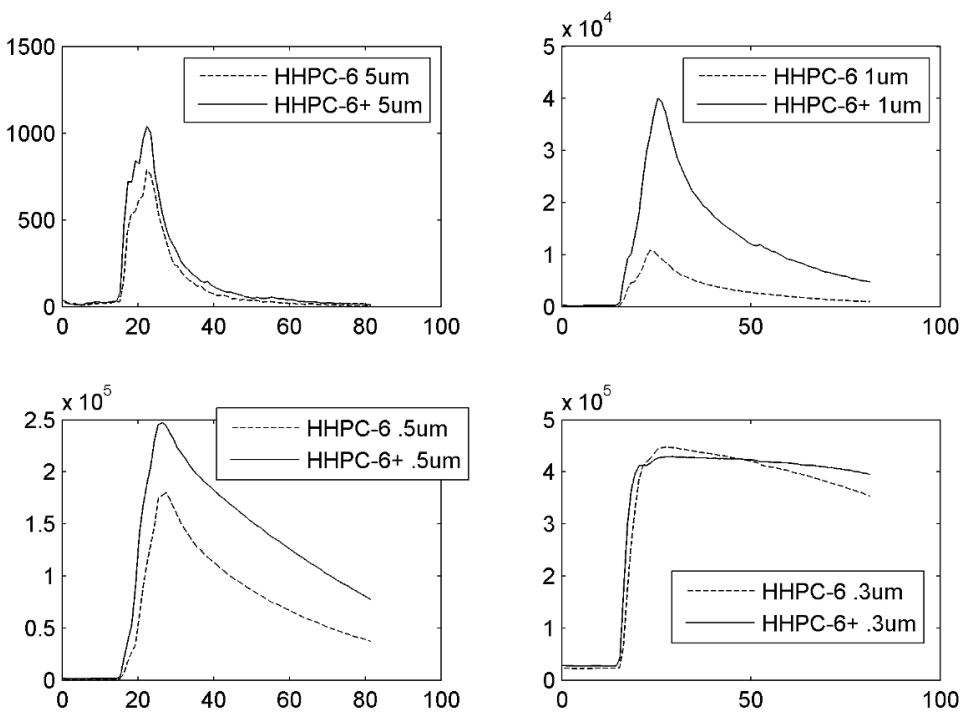

Figure 6: Comparison of additional HHPC size channels for the cooking test, showing ppl vs. time (minutes).

Table 3: Table of cooking $r^{2}$ values. Shaded cells indicate $r^{2}>0.9$.

\begin{tabular}{|c|c|c|c|c|c|c|c|c|c|c|c|c|c|c|}
\hline & & \multicolumn{3}{|c|}{ Specks } & \multicolumn{5}{|c|}{ HHPC-6 } & \multicolumn{5}{|c|}{ HHPC-6+ } \\
\hline & & \begin{tabular}{|l|} 
Speck 1 \\
\end{tabular} & Speck 2 & Speck 3 & $.3 \mathrm{um}$ & $.5 \mathrm{um}$ & $1 \mathrm{um}$ & 2 um & 5 um & 3um & $.5 u m$ & 1 um & $2 \mathrm{um}$ & 5 um \\
\hline \multirow[b]{3}{*}{ Specks } & Speck 1 & 1.000 & 0.987 & 0.994 & 0.193 & 0.567 & 0.955 & 0.966 & 0.796 & 0.156 & 0.498 & 0.863 & 0.956 & 0.726 \\
\hline & Speck 2 & 0.987 & 1.000 & 0.981 & 0.200 & 0.626 & 0.950 & 0.926 & 0.715 & 0.155 & 0.536 & 0.900 & 0.928 & 0.639 \\
\hline & Speck 3 & 0.994 & 0.981 & 1.000 & 0.175 & 0.547 & 0.950 & 0.972 & 0.816 & 0.142 & 0.478 & 0.850 & 0.960 & 0.752 \\
\hline \multirow[b]{5}{*}{ HHPC-6 } & $.3 \mathrm{um}$ & 0.193 & 0.200 & 0.175 & 1.000 & 0.648 & 0.349 & 0.207 & 0.050 & 0.974 & 0.799 & 0.446 & 0.288 & 0.041 \\
\hline & .5um & 0.567 & 0.626 & 0.547 & 0.648 & 1.000 & 0.723 & 0.509 & 0.186 & 0.534 & 0.965 & 0.886 & 0.612 & 0.148 \\
\hline & 1um & 0.955 & 0.950 & 0.950 & 0.349 & 0.723 & 1.000 & 0.950 & 0.702 & 0.296 & \begin{tabular}{l|}
0.679 \\
\end{tabular} & 0.945 & 0.980 & 0.640 \\
\hline & 2um & 0.966 & 0.926 & 0.972 & 0.207 & 0.509 & 0.950 & 1.000 & 0.879 & 0.181 & 0.473 & 0.807 & 0.984 & 0.828 \\
\hline & 5 um & 0.796 & 0.715 & 0.816 & 0.050 & 0.186 & 0.702 & 0.879 & 1.000 & 0.053 & 0.173 & 0.485 & 0.804 & 0.983 \\
\hline \multirow[b]{5}{*}{ HHPC-6+ } & .3um & 0.156 & 0.155 & 0.142 & 0.974 & 0.534 & 0.296 & 0.181 & 0.053 & 1.000 & 0.693 & 0.367 & 0.254 & 0.049 \\
\hline & $.5 u m$ & 0.498 & 0.536 & 0.478 & 0.799 & 0.965 & 0.679 & 0.473 & 0.173 & 0.693 & 1.000 & 0.820 & 0.580 & 0.141 \\
\hline & 1um & 0.863 & 0.900 & 0.850 & 0.446 & 0.886 & 0.945 & 0.807 & 0.485 & 0.367 & 0.820 & 1.000 & 0.875 & 0.425 \\
\hline & 2 um & 0.956 & 0.928 & 0.960 & 0.288 & 0.612 & 0.980 & 0.984 & 0.804 & 0.254 & 0.580 & 0.875 & 1.000 & 0.761 \\
\hline & 5 um & 0.726 & 0.639 & 0.752 & 0.041 & 0.148 & 0.640 & 0.828 & 0.983 & 0.049 & 0.141 & 0.425 & 0.761 & 1.000 \\
\hline
\end{tabular}

sizes remained relatively constant, or that many particles fell within the $1 \mu \mathrm{m}$ to $2 \mu \mathrm{m}$ range.

The incense test was less conclusive, in that each of the five instruments demonstrated much weaker correlation values. The strongest correlations in this experiment were between the $1 \mu \mathrm{m}, 2 \mu \mathrm{m}$, and $5 \mu \mathrm{m}$ channels of the HHPC-6. The similar magnitude in error between the HHPC-6 instruments across both experiments strongly suggests that the instruments differ in calibration. The magnitude of the error between the calibrated test Specks and the HHPC-6+ 


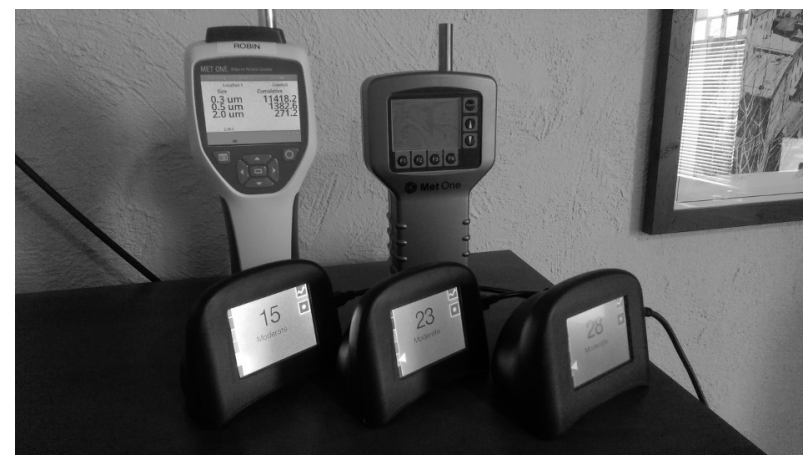

Figure 7: Incense setup. Notice the close spacing of the instruments, which may have disrupted readings (see Results and discussion).

$2 \mu \mathrm{m}$ channel is notably less than the error between the two HHPC units in both experiments. We conclude that the Speck can be calibrated to closely match the performance of handheld optical particle counters intended for the professional market.

Because of the correlation discrepancy between the HHPC instruments in the incense test, we conclude that factors such as airflow and obstructions may have interfered with the even dispersal of particles between the instruments. In future



Figure 8: Plot of incense data over time. 

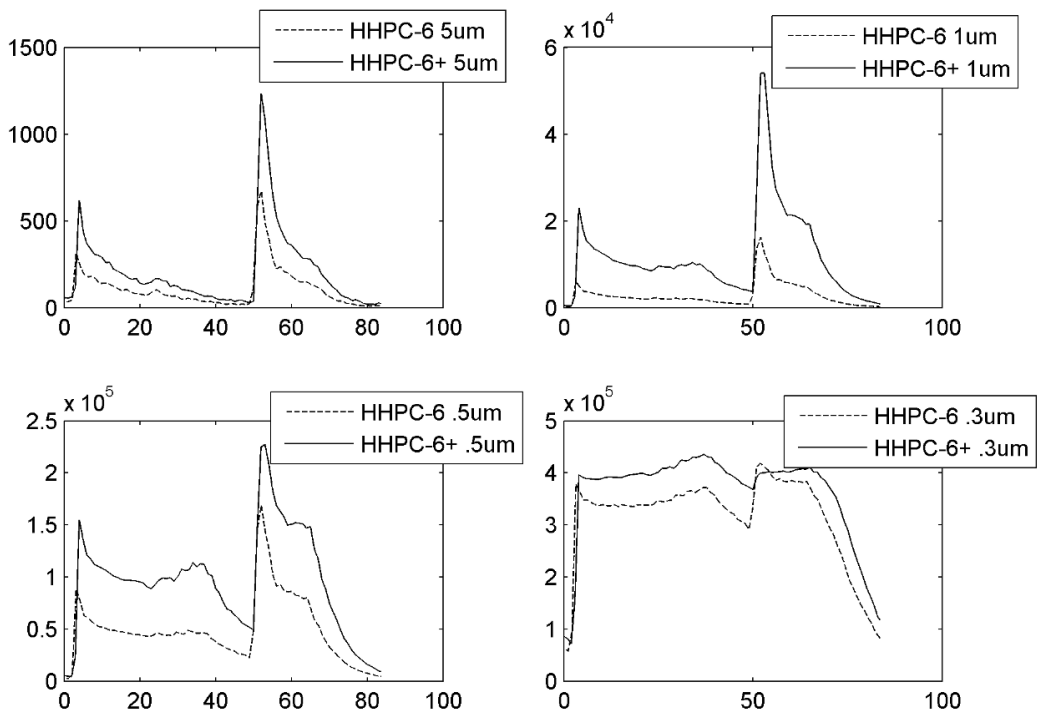

Figure 9: Comparison of additional HHPC size channels for the incense test, showing ppl vs. time (minutes).

Table 4: Table of incense $r^{2}$ values. Shaded cells indicate $r^{2}>0.9$.

\begin{tabular}{|c|c|c|c|c|c|c|c|c|c|c|c|c|c|c|}
\hline & & \multicolumn{3}{|c|}{ Specks } & \multicolumn{5}{|c|}{ HHPC-6 } & \multicolumn{5}{|c|}{ HHPC-6+ } \\
\hline & & Speck 1 & Speck 2 & Speck 3 & $.3 \mathrm{um}$ & $.5 \mathrm{um}$ & $1 \mathrm{um}$ & $2 \mathrm{um}$ & 5 um & $.3 \mathrm{um}$ & $.5 \mathrm{um}$ & $1 \mathrm{um}$ & 2 um & 5 um \\
\hline \multirow[b]{3}{*}{ Specks } & eck 1 & 1.000 & 0.790 & 0.832 & 0.390 & 0.527 & 0.400 & 0.374 & 0.345 & 0.251 & 0.706 & 0.596 & 0.544 & 0.531 \\
\hline & Speck 2 & 0.790 & 50 & 907 & 0.343 & 0.736 & 694 & .670 & 18 & 171 & 0.775 & 8.894 & 874 & 834 \\
\hline & Speck 3 & 0.832 & 907 & 1.000 & 0.341 & 0.651 & 0.592 & 0.575 & 0.557 & 0.186 & 0.720 & 0.774 & 0.751 & 0.751 \\
\hline \multirow[b]{5}{*}{ HHPC- 6} & $.3 \mathrm{um}$ & 390 & 343 & 341 & 1.000 & 0.548 & 322 & 0.279 & 0.250 & 8.858 & 0.727 & 93 & 0.311 & $\overline{0.262}$ \\
\hline & $.5 u m$ & 527 & 736 & 651 & 48 & .000 & 935 & 0.907 & 0.861 & .249 & 0.843 & .907 & 80 & 39 \\
\hline & 1um & 0.400 & 594 & 592 & 0.322 & 0.935 & 1.000 & 0.996 & 0.955 & 0.099 & 0.662 & 0.897 & 19 & 894 \\
\hline & $2 \mathrm{um}$ & 0.374 & & 775 & & 0.907 & 0.996 & 1.000 & 0.972 & 0.073 & 0.613 & 8.871 & & \\
\hline & 5 um & 345 & 18 & 0.557 & 0.250 & 0.861 & 0.955 & 0.972 & 1.000 & 0.053 & 0.554 & 0.810 & 0.849 & 0.899 \\
\hline \multirow[b]{5}{*}{ HHPC-6+ } & 210 & 251 & & 0.186 & 70 & 0.249 & 0,099 & 0.073 & 0.053 & 1000 & 0.500 & .178 & & 88 \\
\hline & $.5 u m$ & 706 & 5 & 720 & 0.727 & 843 & 0.662 & .613 & 0.554 & 0.500 & 1.000 & 0.831 & 0.749 & 0.681 \\
\hline & 1um & 0.596 & 0.894 & 0.774 & 0.393 & 0.907 & 0.897 & 0.871 & 0.810 & 0.178 & 0.831 & 1.000 & 0.989 & 0.938 \\
\hline & $2 \mathrm{um}$ & 0.544 & 0.874 & 0.751 & \begin{tabular}{|l|}
0.311 \\
\end{tabular} & 0.880 & 0.919 & 0.904 & 0.849 & 0.118 & 0.749 & 0.989 & 1.000 & 0.962 \\
\hline & 5 um & 0.531 & 0.834 & 0.751 & 0.262 & 0.839 & 0.894 & 0.896 & 0.899 & 0.080 & 0.681 & 0.938 & 0.962 & 1.000 \\
\hline
\end{tabular}

work, this experiment should be repeated in a more controlled environment, with the instruments closer to the pollutant source and away from obstructions.

Both experiments expose the instruments to very high particle concentrations. The shape of the $0.3 \mu \mathrm{m}$ data from both HHPC instruments and both experiments suggest that the instruments may saturate at levels just under $5 \times 10^{5} \mathrm{ppl}$. Another future experiment should include tests performed at ambient household particle concentrations in order to evaluate the performance of the Speck and 
corresponding calibration values at lower pollution levels. Ideally, calibration can also be performed at these lower concentrations for ease of scalability.

Ultimately, we plan to have the Speck measure particle counts as well as mass concentration in $\mu \mathrm{g} / \mathrm{m}^{3}$, as this is the most common reporting method for $P M_{2.5}$ among state and federal monitoring stations. This would allow users to compare their indoor air quality with the outdoor air quality of their local region. This raises several challenges, however, as our sensor is optical rather than massbased, and typical $P M_{2.5}$ measurements are the product of averaging over one to 24 hours. Additionally, the relationship between particle counts and mass will vary with particle composition. At present, we estimate mass readings using a linear scale factor generated from fitting Speck particle count data to that of a co-located tapered element oscillating microbalance (TEOM) monitor owned by the Allegheny County Health Department. Future works will incorporate onboard humidity and temperature measurements to refine both the particle count accuracy as well as the mass estimate.

\section{References}

[1] Koenig, J. Q., Mar, T. F., Allen, R. W., Jansen, K., Lumley, T., Sullivan, J. H., Trenga, C.A., Larson, T.V., Liu, L. J. S. Pulmonary effects of indoor-and outdoor-generated particles in children with asthma. Environmental Health Perspectives, 113: 499-503. 2005.

[2] Liao, D., Creason, J., Shy, C., Williams, R., Watts, R., Zweidinger, R. Daily variation of particulate air pollution and poor cardiac autonomic control in the elderly. Environmental Health Perspectives, 107: 521-525. 1999.

[3] Pope, C. A., Burnett, R. T., Thurston, G. D., Thun, M. J., Calle, E. E., Krewski, D., Godleski, J. J. Cardiovascular mortality and long-term exposure to particulate air pollution epidemiological evidence of general pathophysiological pathways of disease. Circulation, 109: 71-77. 2004.

[4] Burnett, R.T., Pope, C.A. III, Ezzati, M., Olives, C., Lim, S.S., Mehta, S., Shin, H.H., Singh, G., Hubbell, B., Brauer, M., Anderson, H.R., Smith, K.R., Balmes, J.R., Bruce, N.G., Kan, H., Laden, F., Pruess-Ustuen, A., Turner, M.C., Gapstur, S.M., Diver, W.R., Cohen, A. An integrated risk function for estimating the global burden of disease attributable to ambient fine particulate matter exposure, Environmental Health Perspectives 122: 397-403. 2014.

[5] Litton, C.D. Studies of the measurement of respirable coal dusts and diesel particulate matter, Meas. Sci. Technol. 13: 365-374. 2002.

[6] Kim, E., Hopke, P. K., Pinto, J.P., Wilson, W.E. Spatial variability of fine particle mass, components, and source contributions during the Regional Air Pollution Study in St. Louis. Environ. Sci. Technol. 39: 4172-4179. 2005.

[7] Holstius, D. Monitoring Particulate Matter with Commodity Hardware (Doctoral dissertation, University of California, Berkeley). 2014.

[8] Chantrell, Nathan. Air Quality Egg - Community Air Quality Monitoring, http://nathan.chantrell.net/20130210/air-quality-egg-community-airquality-monitoring/. 
[9] DC1100 Air Quality Monitor, Air Quality, Laser Particle Counter, http://www.dylosproducts.com/learnabout.html.

[10] Hillen, B. Bpeer tracks your home's air quality, http://www.slashgear.com/ bpeer-tracks-your-homes-air-quality-18359937/ 2014.

[11] Air.Air! - Portable Air Dust Detector for Mobile Device, http://www. airair.info/ 2014.

[12] Dust Sensor Module Specifications, http://www.samyoungsnc.com/products/ 3-1 Specification DSM501.pdf. 